\title{
PACHYPHYTUM BREVIFOLIUM ROSE (CRASSULACEAE) A UN SIGLO DE SU DESCUBRIMIENTO Y PACHYPHYTUM GARCIAE, UNA ESPECIE NUEVA DEL CENTRO DE MEXICO
}

\author{
Emmanuel Perez-Calix ${ }^{1}$ \\ Instituto de Ecología, A.C. \\ Centro Regional del Bajío \\ Apartado postal 386 \\ 61600 Pátzcuaro, Michoacán \\ Y \\ Charles Glass ${ }^{2}$
}

\section{RESUMEN}

Se dan a conocer dos localidades del estado de Guanajuato en las que se encontró a Pachyphytum brevifolium Rose; tal especie no se había vuelto a localizar en su ambiente natural desde su descubrimiento por Alfred A. D. Dugès en 1898. Se presenta una descripción y una ilustración de las plantas colectadas y se discute su posible relación con otras especies del género.

Por otro lado, se propone a Pachyphytum garciae como una especie nueva para la ciencia, la nueva entidad recuerda parcialmente a Pachyphytum fittkaui Moran por sus hojas elípticas, elípticooblanceoladas, obovadas a obovado-espatuladas, pero debido a que sus brácteas son de menor tamaño, no imbricadas, así como por la longitud y grueso de sus pedicelos, se separa no sólo de este taxon sino de la sección Ixiocaulon. $P$. garciae también está estrechamente relacionado con $P$. caesium Moran, del que se diferencia en el color de los lóbulos de la corola, así como en la forma y dimensiones de las hojas.

\section{ABSTRACT}

Two localities for Pachyphytum brevifolium from the state of Guanajuato are reported. This species had not been found since its discovery by Alfred A. D. Dugès in 1898. A description of the collected plants is presented and their relationship with other species of the genus is discussed.

Pachyphytum garciae is described as a new taxon. It partially resembles Pachyphytum fittkaui Moran in its elliptic, elliptic-oblanceolate, obovate to obovate-spathulate leaves, but it differs in its smaller, non-imbricate bracts, as well as in the length and thickness of its pedicels, which separate it not only from $P$. fittkaui but also from the section Ixiocaulon. $P$. garciae Moran is also closely related to $P$. caesium, but differs in the color of the lobes of the corolla, as well as in the form and dimension of the leaves.

\footnotetext{
${ }^{1}$ Trabajo realizado con apoyo económico del Instituto de Ecología, A.C. (cuenta 902-03), del Consejo Nacional de Ciencia y Tecnología y de la Comisión Nacional para el Conocimiento y Uso de la Biodiversidad.

${ }^{2}$ C. Glass, falleció el día 23 de febrero de 1998.
} 


\section{Pachyphytum brevifolium a un siglo de su descubrimiento}

Pachyphytum brevifolium Rose está descrito en la parte correspondiente a la familia Crassulaceae de la Flora de Norteamérica (Britton y Rose, 1905); su diagnosis se basó en plantas colectadas por Alfred A. D. Dugès en enero de 1898, pero infortunadamente la localidad señalada en la etiqueta que acompaña al ejemplar dice sólo "near Guanajuato", por lo que se desconoce el lugar preciso de donde es originario, y no se ha vuelto a encontrar por más de 100 años.

Aunque se cuenta con el ejemplar tipo, depositado en el herbario de la Universidad de Harvard $(\mathrm{GH})$, así como con un fragmento de la planta de un ejemplar localizado en el Herbario Nacional de los Estados Unidos de América (US), la escasez de material originario de la región donde fue colectado ha traído como consecuencia el uso incorrecto del nombre. Tales son los casos de Walther (1932) y de von Poellnitz (1937), quienes determinaron equivocadamente como $P$. brevifolium una planta que más tarde Moran (1963) describió como Pachyphytum glutinicaule. Otro efecto de no contar con material proveniente de las cercanías de la localidad en la que se colectó el tipo es que no se han logrado definir sus relaciones con las demás especies del género (Moran, 1963, 1968, 1971, 1991). Meyrán (1997) presenta una descripción de la especie en cuestión, en base a plantas cultivadas. Según su apreciación $P$. brevifolium pertenece a la sección Pachyphytum.

En exploraciones recientes realizadas en el municipio de Guanajuato se encontraron dos poblaciones de plantas cuyas características corresponden a Pachyphytum brevifolium. A continuación se presenta una descripción basada en los ejemplares colectados.

Pachyphytum brevifolium Rose, North Amer. Flora 22: 12. 1905. Fig. 1.

Nombre común registrado en la región: dedito de dios.

Planta herbácea perenne a sufrútice, postrada, decumbente o colgante, llegando a formar colonias densas; tallo simple o ramificado, de hasta $1 \mathrm{~m}$ de largo; hojas dispuestas en espiral, agrupadas hacia el ápice del tallo, ocasionalmente formando una roseta, láminas elípticas a estrechamente obovadas en contorno, oblatas a estrechamente elípticas en sección transversal, ápice redondeado a ampliamente obtuso, de 1 a $3 \mathrm{~cm}$ de largo por 5 a $10 \mathrm{~mm}$ de ancho y 3 a $8 \mathrm{~mm}$ de grueso, de color verde oscuro, ocasionalmente caférojizo, glaucas, con una mancha roja oscura en el ápice; inflorescencia en forma de cincino, pedúnculo de 6.0 a $8.5 \mathrm{~cm}$ de largo, con brácteas dispuestas en espiral, de 6 a $8 \mathrm{~mm}$ de largo, 2 a $3 \mathrm{~mm}$ de ancho y ca. $1 \mathrm{~mm}$ de grueso, elíptico-ovadas, brácteas del cincino no imbricadas, elíptico-ovadas, agudas, de base truncada, de 5 a $9 \mathrm{~mm}$ de largo, 2 a $3 \mathrm{~mm}$ de ancho, pedicelos de 4 a $15 \mathrm{~mm}$ de largo, rojizos; cáliz de 6 a $7 \mathrm{~mm}$ de largo, tan largo o ligeramente (1 a $3 \mathrm{~mm}$ ) más corto que la corola, segmentos erectos y adpresos a la corola, elíptico-ovados, obtusos a redondeados, cortamente mucronados, de 5 a $7 \mathrm{~mm}$ de largo, de ca. $2 \mathrm{~mm}$ de ancho, glaucos, de color rosado-anaranjado a rojo claro, el ápice verdoso; corola subcampanulada, segmentos connados muy cerca de la base, oblongo-oblanceolados, obtusos, apiculados, de 6 a $7 \mathrm{~mm}$ de largo, de 2 a $3 \mathrm{~mm}$ de ancho, glaucos, apéndices escamosos de ca. $1.5 \mathrm{~mm}$ de largo, de color amarillo; glándulas nectaríferas de ca. $1 \mathrm{~mm}$ de ancho, amarillas; carpelos erectos, adpresos, de 3 a $5.5 \mathrm{~mm}$ de largo, estilos de $1 \mathrm{~mm}$ de largo, óvulos numerosos; folículos de dehiscencia longitudinal. 
Pérez-Calix y Glass: Pachyphytum brevifolium A Un Siglo de su Descubrimiento y P. garciae

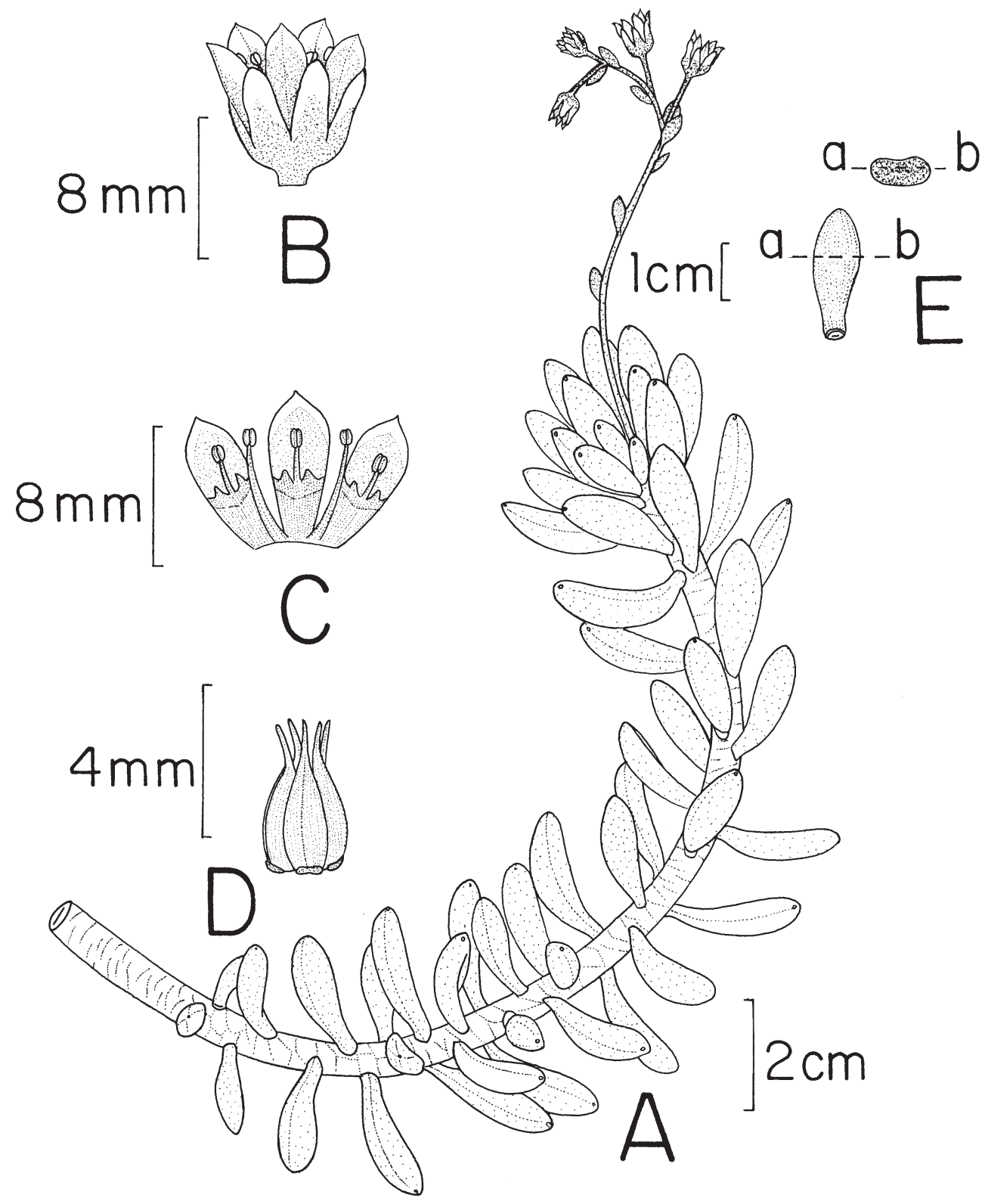

Fig. 1. Pachyphytum brevifolium Rose. A. hábito de la planta; B. flor; C. vista ventral de los segmentos de la corola; D. carpelos y nectarios; E. hoja en vista ventral; a-b. sección transversal de la hoja. (Dibujo realizado por Rogelio Cárdenas). 
TIPO: near Guanajuato, Mexico, jan. 1898, A. Dugès 153 (holotipo: GH; isotipo: US!)

Distribución: Considerando que no se puede definir el sitio en que A. Dugès realizó su colecta, Pachyphytum brevifolium se conoce únicamente de las siguientes localidades:

Guanajuato: cascada cerca de Picones, municipio de Guanajuato, 2150 m s.n.m., E. Pérez y E. Carranza 2875 (IEB); alrededores de Picones, cerca del río, municipio de Guanajuato, 2150 m s.n.m., E. Pérez y C. Glass 3793 (IEB); Cañada del Capulín, cerca de La Sauceda, municipio de Guanajuato, 2000 m s.n.m., E. Pérez y C. Glass 3796 (IEB).

En la región la vegetación predominante es el bosque de encino; Pachyphytum brevifolium crece en paredes de rocas ígneas, formando colonias densas o como individuos aislados. Florece en (diciembre) enero y febrero (marzo).

En la zona se usa como ornamental, particularmente en los festejos de Navidad.

En la descripción que aquí se ofrece se aprecian algunas diferencias con relación a las de Rose (1905) y Moran (1963), ambas elaboradas a partir del ejemplar tipo. De acuerdo con los autores mencionados, $P$. brevifolium presenta un tallo de 4 a 5 o más cm de largo, un pedúnculo de $2 \mathrm{~cm}$ de largo y las flores un pedicelo de 4 a $5 \mathrm{~mm}$ de largo; mientras que las plantas observadas por nosotros presentan tallos de hasta $1 \mathrm{~m}$ de largo, pedúnculo de 6 a $8.5 \mathrm{~cm}$ de largo y pedicelos de 4 a $15 \mathrm{~mm}$ de largo. Tanto J. N. Rose como R. Moran basaron su descripción en la misma y única planta, por lo que las diferencias anotadas se pueden considerar como parte de la variación natural de la especie en cuestión.

Por sus hojas menos de dos veces más anchas que gruesas, sus brácteas pequeñas, no estrechamente imbricadas en el cincino, así como porque los lóbulos de la corola son uniformemente coloreados, $P$. brevifolium se ubica en la sección Diotostemon (Salm-Dyck) Walther, junto con Pachyphytum compactum Rose, $P$. coeruleum Meyran y P. hookeri (SalmDyck) A. Berger. De estas últimas se diferencia por los segmentos del cáliz que son casi tan largos o ligeramente (1 a $3 \mathrm{~mm}$ ) más cortos que la corola, elíptico-ovados, con el ápice obtuso a redondeado y se relaciona mejor con las especies de la sección Ixiocaulon ( $P$. fittkaui Moran y $P$. glutinicaule Moran), aunque las hojas, las brácteas, los pedicelos, además del hábito de esos taxa son diferentes a tales estructuras en $P$. brevifolium (Cuadro 1). Por otro lado, de las especies que forman la sección Pachyphytum se diferencia tanto en su hábito, como en la forma de las hojas, además del aspecto general de la inflorescencia. Acorde con lo anterior, Pachyphytum brevifolium parece ser intermedio entre las especies de la sección Diotostemon y las de Ixiocaulon (Cuadro 1).

Pachyphytum garciae, una especie nueva del centro de México

Durante una exploración realizada en la zona noreste de Guanajuato y la región vecina de Querétaro por el personal del Jardín Botánico "El Charco del Ingenio" (CANTE, A.C.), el joven Juan Antonio García Luna encontró una especie de Pachyphytum diferente a las ya conocidas, por lo que se propone aquí como nueva para la ciencia, cuyo nombre a petición del segundo autor, se dedica a su descubridor.

Pachyphytum garciae Pérez-Calix et Glass sp. nov. Fig. 2.

Planta subfrutescens aliquantum glauca dependens vel decumbens; caules usque 40 cm longi; folia in rosulis basalis disposita, elliptico-oblanceolata, obovata vel obovato- 
Cuadro 1. Comparación entre las características de las secciones reconocidas de Pachyphytum frente a las de $P$. brevifolium y $P$. garciae.

\begin{tabular}{|c|c|c|c|c|c|}
\hline Sección & Diotostemon & Pachyphytum & Ixiocaulon & & \\
\hline Especies & $\begin{array}{l}P . \text { compactum } \\
P . \text { hookeri } \\
P . \text { coeruleum }\end{array}$ & $\begin{array}{l}P . \text { bracteosum } \\
P . \text { kimnachi } \\
P . \text { longifolium } \\
P . \text { oviferum } \\
P . \text { viride } \\
P . \text { werdermanii }\end{array}$ & $\begin{array}{l}\text { P. fittkaui } \\
\text { P. glutinicaule }\end{array}$ & P. brevifolium & P. garciae \\
\hline Hojas & $\begin{array}{l}\text { subrollizas y más o me } \\
\text { nos fusiformes }\end{array}$ & $\begin{array}{l}\text { elípticas a obovadas; me } \\
\text { nos de dos a cinco ve- } \\
\text { ces más anchas que } \\
\text { gruesas }\end{array}$ & $\begin{array}{l}\text { obovado a espatuladas; } \\
\text { dos o más veces más } \\
\text { anchas que gruesas }\end{array}$ & $\begin{array}{l}\text { elípticas a estrechamen- } \\
\text { te obovadas; ligeramen- } \\
\text { te más anchas que grue } \\
\text { sas a cerca de dos ve } \\
\text { ces más anchas que } \\
\text { gruesas }\end{array}$ & $\begin{array}{l}\text { elípticas, elíptico-lanceo } \\
\text { ladas, obovadas a es- } \\
\text { patuladas; dos o más ve } \\
\text { ces más anchas que } \\
\text { gruesas }\end{array}$ \\
\hline $\begin{array}{l}\text { Acomodo de las brác- } \\
\text { teas en el cincino joven }\end{array}$ & $\begin{array}{l}\text { separadas y no imbrica- } \\
\text { das }\end{array}$ & $\begin{array}{l}\text { estrechamente imbrica- } \\
\text { das }\end{array}$ & $\begin{array}{l}\text { estrechamente imbrica- } \\
\text { das }\end{array}$ & parcialmente imbricadas & parcialmente imbricadas \\
\hline Base de las brácteas & entera a bidentada & $\begin{array}{l}\text { sagitado-abrazadora a } \\
\text { bidentada }\end{array}$ & subtruncada a sagitada & truncada & entera \\
\hline $\begin{array}{l}\text { Largo de los pedicelos } \\
(\mathrm{mm})\end{array}$ & 5 a $20(35)$ & 2 a 8 & 2 a 15 & 4 a 15 & 3 a 8 \\
\hline Relación corola-cáliz & $\begin{array}{l}\text { cáliz de ca. de la mitad } \\
\text { de longitud de la corola }\end{array}$ & $\begin{array}{l}\text { corola más corta que } \\
\text { el cáliz }\end{array}$ & $\begin{array}{l}\text { cáliz tan largo o ligera- } \\
\text { mente más corto que la } \\
\text { corola }\end{array}$ & $\begin{array}{l}\text { cáliz tan largo o ligera- } \\
\text { mente más corto que la } \\
\text { corola }\end{array}$ & $\begin{array}{l}\text { cáliz tan largo o ligera- } \\
\text { mente más corto que la } \\
\text { corola }\end{array}$ \\
\hline Segmentos del cáliz & $\begin{array}{l}\text { subiguales en largo y muy } \\
\text { poco diferentes en an- } \\
\text { cho, ligeramente imbrica } \\
\text { dos en el botón muy jo } \\
\text { ven, después todos uni- } \\
\text { formemente separados }\end{array}$ & $\begin{array}{l}\text { marcadamente desigua- } \\
\text { les en dimensiones y for } \\
\text { ma, } 3 \text { mayores estre- } \\
\text { chamente imbricados, } 2 \\
\text { pequeños comúnmente } \\
\text { abiertos y no imbricados } \\
\text { con los } 3 \text { mayores }\end{array}$ & $\begin{array}{l}\text { marcadamente desigua- } \\
\text { les en longitud y forma, } \\
3 \text { mayores imbricados en } \\
\text { botón pero se abren des } \\
\text { pués de la antesis y fre } \\
\text { cuentemente separados } \\
\text { inclusive en el botón jo- } \\
\text { ven }\end{array}$ & $\begin{array}{l}\text { desiguales en longitud y } \\
\text { forma }\end{array}$ & $\begin{array}{l}\text { desiguales en longitud y } \\
\text { forma, } 2 \text { mayores en an- } \\
\text { cho y ligeramente en lar } \\
\text { go, opuestos entre ellos, } \\
\text { imbricados a los otros } \\
\text { en el botón, pero separa } \\
\text { dos en la antesis }\end{array}$ \\
\hline Segmentos de la corola & $\begin{array}{l}\text { permanecen erectos a } \\
\text { lo largo de la antesis }\end{array}$ & $\begin{array}{l}\text { ligeramente recurvados } \\
\text { al final de la antesis }\end{array}$ & $\begin{array}{l}\text { recurvados al final de la } \\
\text { antesis }\end{array}$ & $\begin{array}{l}\text { extendidos a ligeramente } \\
\text { recurvados al final de la } \\
\text { antesis }\end{array}$ & $\begin{array}{l}\text { extendidos a ligeramen- } \\
\text { te recurvados al final de } \\
\text { la antesis }\end{array}$ \\
\hline $\begin{array}{l}\text { Color de los lóbulos de } \\
\text { la corola }\end{array}$ & $\begin{array}{l}\text { uniformemente colorea- } \\
\text { dos, o bien, con un co- } \\
\text { lor diferente en el ápice } \\
\text { de los segmentos, pero } \\
\text { sin una mancha oscura } \\
\text { en la región ventral }\end{array}$ & $\begin{array}{l}\text { blanco a rosado pero } \\
\text { con una mancha oscu- } \\
\text { ra en la región media- } \\
\text { ventral y cubriendo las } \\
\text { escamas petaloideas }\end{array}$ & $\begin{array}{l}\text { rosa oscuro, uniforme } \\
\text { mente coloreados u os } \\
\text { cureciéndose ventralmen } \\
\text { te y con los ápices lige } \\
\text { ramente más claros }\end{array}$ & $\begin{array}{l}\text { rosa-anaranjado a rojo } \\
\text { claro, ápice verdoso }\end{array}$ & $\begin{array}{l}\text { rosa claro, oscurecién- } \\
\text { dose ligeramente en la } \\
\text { región media }\end{array}$ \\
\hline
\end{tabular}


spathulata, $1.5-4.5 \mathrm{~cm}$ longa, $0.8-2.3 \mathrm{~cm}$ lata, $5-7 \mathrm{~mm}$ crassa, apice late acuta ad rotundata, mucronulata; inflorescentia cincinnalis, pedunculus usque $20 \mathrm{~cm}$ longus, bracteis obovatis vel ellipticis, 6-11 mm longis, 2-6 mm latis, rotundatis vel obtusis, bracteae florales 7-10 mm longae, 4-6 mm latae, pedicelli 3-8.5 mm longi; calycis segmenta oblonga, 6.5-9.5 mm longa, longitudine subaequalia, latitudine inaequalia, duo 3-5 mm lata, inter tria 2-3.5 mm lata alternantia, apice rotundata usque ad acuta; corollae segmenta oblongo-oblanceolata, $8 \mathrm{~mm}$ longa, 2.5-3 mm lata, apice acuta aliquantum apiculata; filamenta antipetala $3 \mathrm{~mm}$ longa, filamenta antisepala $10 \mathrm{~mm}$ longa; nectaria ca. $1 \mathrm{~mm}$ lata; carpella ca. $4 \mathrm{~mm}$ longa; fructus follicularis.

Planta sufrutescente, suculenta, glabra, ligeramente glauca, colgante o decumbente; tallos simples o ramificados desde cerca de la base, hasta de $40 \mathrm{~cm}$ de largo y de $6 \mathrm{~mm}$ o menos de diámetro; hojas dispuestas en rosetas de 5 a $8 \mathrm{~cm}$ de diámetro, laxas o compactas cerca del ápice de las ramas, láminas elípticas, elíptico-oblanceoladas, obovadas a obovado-espatuladas, de 1.5 a $4.5 \mathrm{~cm}$ de largo por 0.8 a $2.3 \mathrm{~cm}$ de ancho y 5 a $7 \mathrm{~mm}$ de grosor, de color verde-azulado, glaucas, ápice ampliamente agudo a redondeado, mucronulado, generalmente teñido de color púrpura; inflorescencia en forma de cincino, pedúnculo de hasta $20 \mathrm{~cm}$ de largo, (incluyendo la inflorescencia), brácteas alternas, obovadas o elípticas, de 6 a $11 \mathrm{~mm}$ de largo por 2 a $6 \mathrm{~mm}$ de ancho, redondeadas a obtusas, ligeramente apiculadas, brácteas florales de 7 a $10 \mathrm{~mm}$ de largo por 4 a $6 \mathrm{~mm}$ de ancho, pedicelos de 3 a $8.5 \mathrm{~mm}$ de largo y $1 \mathrm{~mm}$ de diámetro, de color rosa; cáliz de 5 segmentos fusionados en la base formando una copa de alrededor de $1.5 \mathrm{~mm}$ de alto y cerca de $5 \mathrm{~mm}$ de diámetro, segmentos oblongos, subiguales en largo, de 6.5 a $9.5 \mathrm{~mm}$, evidentemente desiguales en amplitud, dos más anchos, de 3 a $5 \mathrm{~mm}$, alternándose entre 3 más angostos, éstos de 2 a $3.5 \mathrm{~mm}$, ápice redondeado a obtuso; corola de 5 segmentos connados en la base en menos de $0.5 \mathrm{~mm}$, lóbulos oblongo-oblanceolados, de $8 \mathrm{~mm}$ de largo por 2.5 a $3 \mathrm{~mm}$ de ancho, ápice agudo, ligeramente apiculado, cada lóbulo con un par de apéndices escamosos de ca. $1 \mathrm{~mm}$ de largo por ca. $0.5 \mathrm{~mm}$ de ancho, segmentos de color rosa claro, que en la región central se oscurecen ligeramente, amarillentos en la base; filamentos antepétalos de $3 \mathrm{~mm}$ de largo y los antesépalos de $10 \mathrm{~mm}$, de color amarillo en la base y rosa en el ápice; nectarios de ca. $1 \mathrm{~mm}$ de ancho; carpelos de ca. $4 \mathrm{~mm}$ de largo, de color amarillo, estilos de ca. $1 \mathrm{~mm}$, de color rosa; folículos con numerosas semillas.

TIPO: México, Querétaro. El Zapote, \pm 4 km al NW de Río Blanco, municipio de Peñamiller, alt. $1600 \mathrm{~m}$, cañada con matorral submontano, crece en los riscos de rocas calizas. 4.Il.1997, E. Pérez y S. Zamudio 3574 (IEB).

Material adicional revisado: Querétaro. El Zapote, \pm 4 km de Río Blanco, municipio de Peñamiller, E. Pérez y E. Carranza 3798 (IEB).

Pachyphytum garciae se conoce únicamente de las paredes en las que se colectó el tipo, por lo que se considera un endemismo muy estrecho. La zona está formada por rocas calizas del Cretácico; la vegetación que prospera corresponde al matorral submontano, aunque sobre los peñascos en los que vive la planta en cuestión predominan manchones de matorral rosetófilo. 
Pérez-Calix y Glass: Pachyphytum brevifolium A Un Siglo de su Descubrimiento y $P$. garciae
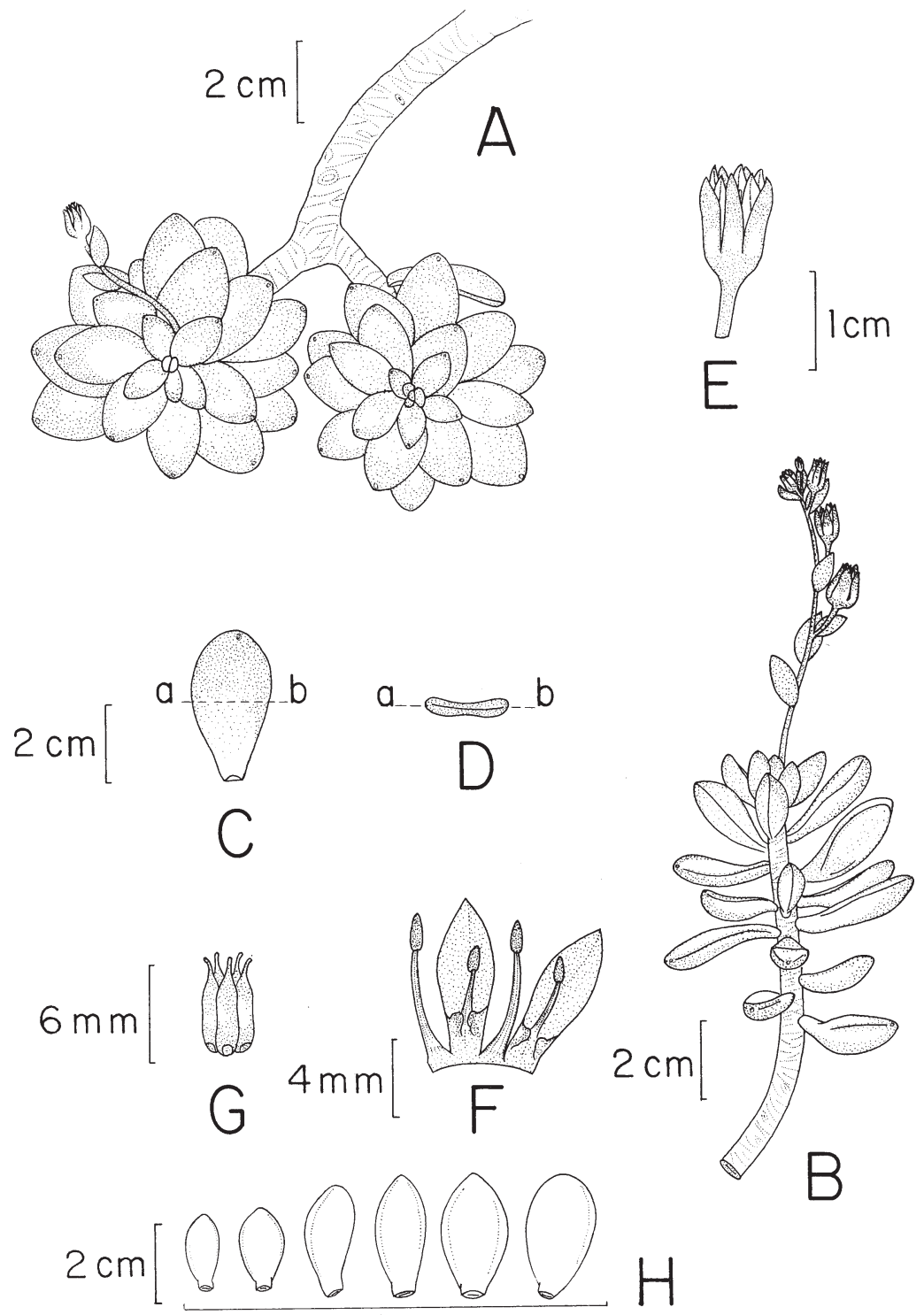

Fig. 2. Pachyphytum garciae Pérez-Calix et Glass sp. nov. A. hábito de la planta; B. rama con inflorescencia; C. hoja en vista ventral; D. corte transversal de la hoja; E. flor; F. vista ventral de los segmentos de la corola; G. carpelos y nectarios; H. variación de las hojas. (Dibujo realizado por Rogelio Cárdenas). 
Se ha observado en floración y fructificación de febrero a abril. Se encuentra en forma vegetativa durante todo el año, a lo largo del cual se pueden apreciar sus rosetas, formadas por las hojas, sobre los tallos colgantes, en los riscos donde crece.

Las hojas elípticas, elíptico-oblanceoladas, obovadas a obovado-espatuladas, dos o más veces más anchas que gruesas relacionan a la nueva entidad con $P$. fittkaui Moran (sección Ixiocaulon). Sin embargo, las brácteas del cincino no estrechamente imbricadas y de menores dimensiones, el largo y grueso de los pedicelos, la combinación de colores en la corola, así como los segmentos del cáliz de menores dimensiones, separan a $P$. garciae de los elementos de la mencionada sección (Cuadros 1 y 2).

Cuadro 2. Comparación de Pachyphytum garciae con las especies más relacionadas.

\begin{tabular}{|c|c|c|c|}
\hline & P. caesium & P. garciae & P. fittkaui \\
\hline $\begin{array}{l}\text { Tallo } \\
\text { largo }(\mathrm{cm}) \\
\text { diámetro }(\mathrm{cm})\end{array}$ & $\begin{array}{l}30 \text { o más } \\
\text { (1) } 1.5 \text { a } 2.1\end{array}$ & $\begin{array}{l}\text { hasta } 40 \\
0.6 \text { o menos }\end{array}$ & $\begin{array}{l}\text { hasta } 100 \text { o más } \\
1.5(3.5)\end{array}$ \\
\hline $\begin{array}{l}\text { Hojas } \\
\text { forma }\end{array}$ & obovado-oblonga & $\begin{array}{l}\text { elíptica, elíptico-lanceo } \\
\text { lada, obovada a espa- } \\
\text { tulada }\end{array}$ & $\begin{array}{l}\text { elíptica a elíptico-oblan } \\
\text { ceolada a obovada }\end{array}$ \\
\hline $\begin{array}{l}\text { largo }(\mathrm{cm}) \\
\text { ancho }(\mathrm{cm}) \\
\text { grueso }(\mathrm{cm})\end{array}$ & $\begin{array}{l}3 \text { a } 5(6.5) \\
(1) 2 \text { a } 3.6 \\
0.6 \text { a } 1.4\end{array}$ & $\begin{array}{l}1.5 \text { a } 4.5 \\
0.8 \text { a } 2.3 \\
0.5 \text { a } 0.7\end{array}$ & $\begin{array}{l}3 \text { a } 9.5 \\
1.5 \text { a } 3.5 \\
0.9 \text { a } 1.5\end{array}$ \\
\hline $\begin{array}{l}\text { Brácteas florales } \\
\text { forma } \\
\text { largo }(\mathrm{mm}) \\
\text { ancho }(\mathrm{mm})\end{array}$ & $\begin{array}{l}\text { elíptica } \\
\pm 9 \\
5\end{array}$ & $\begin{array}{l}\text { obovada a elíptica } \\
7 \text { a } 10 \\
4 \text { a } 6\end{array}$ & $\begin{array}{l}\text { ovada } \\
12 \text { a } 25 \\
5 \text { a } 15\end{array}$ \\
\hline $\begin{array}{l}\text { Acomodo de las brácteas } \\
\text { en el cincino joven }\end{array}$ & subimbricadas & subimbricadas & $\begin{array}{l}\text { estrechamente imbrica- } \\
\text { das }\end{array}$ \\
\hline $\begin{array}{l}\text { Pedicelo } \\
\text { largo (mm) } \\
\text { diámetro }(\mathrm{mm})\end{array}$ & $\begin{array}{l}3 \text { a } 5(18) \\
1.5\end{array}$ & $\begin{array}{l}3 \text { a } 8.5 \\
1\end{array}$ & $\begin{array}{l}2 \text { a } 7 \\
2 \text { a } 3.5\end{array}$ \\
\hline $\begin{array}{l}\text { Lóbulos del cáliz } \\
\text { Mayores } \\
\text { largo }(\mathrm{mm}) \\
\text { ancho }(\mathrm{mm}) \\
\text { Menores } \\
\text { largo }(\mathrm{mm}) \\
\text { ancho }(\mathrm{mm})\end{array}$ & $\begin{array}{l}9 \\
6 \\
7 \\
2\end{array}$ & $\begin{array}{l}6.5 \text { a } 9.5 \\
3 \text { a } 5 \\
2 \text { a } 3.5\end{array}$ & $\begin{array}{l}10 \text { a } 21 \\
5 \text { a } 11 \\
7 \text { a } 13 \\
3 \text { a } 6\end{array}$ \\
\hline
\end{tabular}


Cuadro 2. Continuación.

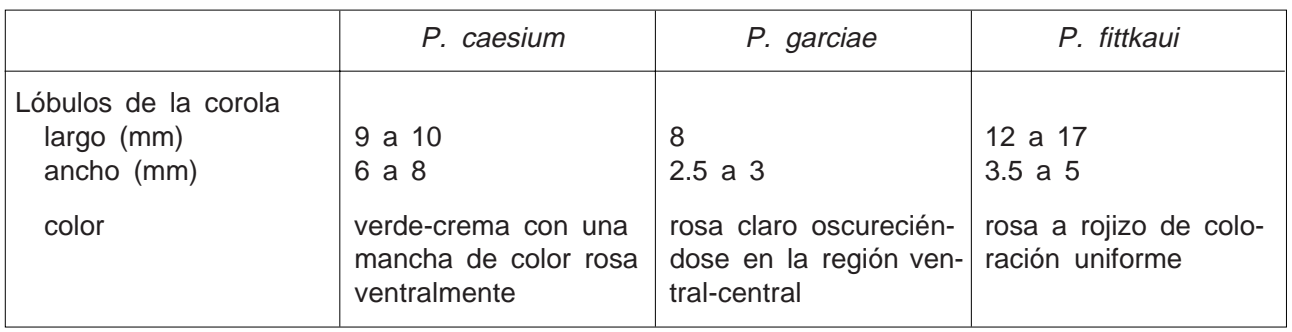

Una especie afín a la descrita aquí es $P$. caesium Moran et Kimnach, taxon descrito de Aguascalientes que habita en una región de rocas volcánicas. Pachyphytum garciae es una planta menos robusta, con hojas elípticas, elíptico-oblanceoladas, obovadas a obovadoespatuladas, con los lóbulos de la corola de color rosa claro oscureciéndose ligeramente en la región ventral; mientras que las hojas de Pachyphytum caesium son ovado-oblongas, y los segmentos de la corola son de color verde-crema con una mancha de color rosa ventralmente (Cuadro 2).

Acorde con lo observado para $P$. brevifolium y $P$. garciae, así como lo discutido por Moran y Kimnach (1993) para $P$. caesium, y García et al. (1999) para $P$. machucae se concluye que el género Pachyphytum requiere con urgencia de un estudio a la luz de los descubrimientos recientes, ya que algunas de las especies no se pueden ubicar de manera adecuada en las secciones previamente propuestas.

\section{AGRADECIMIENTOS}

Manifestamos nuestra sincera gratitud al Dr. Jerzy Rzedowski R. por la traducción de la diagnosis de Pachyphytum garciae al latín, a él mismo, a la maestra Graciela Calderón de Rzedowski y a la Biól. Rosa María Murillo la revisión crítica del manuscrito; a los Bióls. Eleazar Carranza y Sergio Zamudio su ayuda en el trabajo de colecta.

\section{LITERATURA CITADA}

Britton, N. L. \& J. N. Rose. 1905. Crassulaceae. North Am. Flora 22: 7-74.

García, I., C. Glass y M. Cházaro. 1999. Pachyphytum machucae (Crassulaceae) una nueva especie de Michoacán, México. Acta Bot. Mex. 47: 9-14.

Meyrán, J. 1997. Pachyphytum brevifolium Rose. Cact. Suc. Mex. 42: 31-34.

Moran, R. 1963. Pachyphytum brevifolium Rose and Pachyphytum glutinicaule, a new species from Hidalgo, México. Cact. Succ. Jour. (US) 35: 35-41.

Moran, R. 1968. New subgeneric groups in Echeveria and Pachyphytum. Cact. Succ. Jour. (US) 40: 36-42.

Moran, R. 1971. Pachyphytum fittkaui, a new species from Guanajuato, México. Cact. Succ. Jour. (US) 43: 26-32. 
Acta Botanica Mexicana (1999), 48:1-10

Moran, R. 1991. Pachyphytum compactum Rose (Crassulaceae). Cact. Succ. Jour. (US) 63: 30-34.

Moran, R. \& M. Kimnach. 1993. Pachyphytum caesium, a new species from Aguascalientes, México. Cact. Succ. Jour. (US) 65: 59-62.

von Poellnitz, K. 1937. The genus Pachyphytum. Cact. Jour. 5: 72-75.

Walther, E. 1932. Pachyphytum brevifolium. Cact. Succ. Jour. (US) 4: 237.

Aceptado para publicación en agosto de 1999. 